\title{
Patrones Morfológicos Erróneamente Diagnosticados como Huellas de Mordedura en Contextos Forenses: Una Revisión con Búsqueda Sistemática
}

\author{
Morphological Patterns Misdiagnosed as Bite Marks In Forensic Contexts: A Scoping Review
}

Francisca Dal Santo-Mendoza ${ }^{1}$ \& Gabriel M. Fonseca ${ }^{2}$

DAL SANTO-MENDOZA, F. \& FONSECA, G. M. Patrones morfológicos erróneamente diagnosticados como huellas de mordedura en contextos forenses: una revisión con búsqueda sistemática. Int. J. Morphol., 39(2):642-652, 2021.

RESUMEN: El análisis de huellas de mordedura para identificación forense puede ser clave para establecer la inocencia o culpabilidad del acusado. Sin embargo, esta evidencia ha sido cuestionada por su falta de consenso y objetividad, y por sus reportadas identificaciones erróneas. Se presenta una revisión con búsqueda sistemática de casos indicando errores diagnósticos y sus caracterizaciones, y se discuten las recomendaciones realizadas para evitarlos. Se utilizó la estrategia ("bite mark" OR "bitemark")AND ("artefactual" OR "misidentification" OR "misdiagnosis" OR "mistake"), incluyendo casos de huellas de mordedura humana en piel con reportes de errores diagnósticos, excluyendo diseños experimentales, huellas en alimentos u objetos. Fueron seleccionados un total de 13 documentos reportando 21 casos, abarcando los años 1989 a 2014. Los patrones morfológicos con mayor reporte de error diagnóstico fueron los producidos por trauma cortante o contuso por otras causas (10 casos), seguidos de los producidos por condiciones médicas y tratamientos de emergencia (6 casos). Al menos tres de los casos tomaron estado público, dos de ellos con sentencia de muerte y uno con condena de 25 años a prisión perpetua. Se ha enfatizado el dar minuciosidad y estandarización a la evaluación morfológica de este tipo de evidencias por la importante cantidad de condenas erróneas. Esta revisión pone en evidencia la escasa exposición y análisis de estos errores, con la insuficiente información para un aprendizaje significativo crítico y diseño de herramientas para evitarlos. Se coincide en la necesidad de estandarizar y optimizar protocolos para estas evidencias y adoptar una conducta reflexiva para sus análisis con fines de identificación forense.

PALABRAS CLAVE: Error diagnóstico; Huellas de mordedura; Odontología Forense.

\section{INTRODUCCIÓN}

La identificación mediante análisis de huellas de mordedura es uno de los aspectos más desafiantes y discutibles de la odontología forense, ya que este tipo de evidencia puede ser factor clave para exonerar o condenar a un individuo en casos criminales (Reesu \& Brown, 2016). La definición de "análisis" refiere a un estudio cuidadoso de algo para aprender sobre sus partes, lo que hacen y cómo se relacionan entre sí, concepto que en el área de las huellas de mordedura debe ser realizado como parte de la autopsia medicolegal a través de la documentación e interpretación objetiva de la morfología lesional, "sean o no huellas de mordedura" (Souviron \& Haller, 2017).

Una huella de mordedura es definida como una alteración física registrada en un medio, causada por el contacto de dientes humanos o animales (American Board of Forensic Odontology, 2018). Estas huellas forman parte de un tipo es- pecial de lesiones denominadas "lesiones patrón" o "figuradas", lo que significa que la marca observada refleja la forma física y las características del objeto que las produjo (Bórquez, 2012; Forrest \& Soon, 2016). En contextos forenses, y al igual que con otros tipos de pruebas físicas, el análisis de estas huellas de mordedura implica utilizar protocolos y terminología apropiados; es así que las denominadas "características de clase" van a permitir expresar la confianza de que un determinado tipo de objeto (en este caso los dientes) han originado ese patrón, mientras que las "características individuales" son aquellas que permitirán hacer lo propio con una dentición específica y de ahí, identificar al perpetrador (Forrest \& Soon). Este último proceso en particular, ha suscitado una no menor controversia considerando que actualmente no se dispone de información suficiente para aseverar el carácter de "únicos" a los patrones dentarios, o para declarar la posibilidad de que una coincidencia sea solo resultado del azar (Forrest \& Soon).

\footnotetext{
${ }^{1}$ Carrera de Odontología, Facultad de Odontología, Universidad de La Frontera, Temuco, Chile.

${ }^{2}$ Centro de Investigación en Odontología Legal y Forense (CIO), Facultad de Odontología, Universidad de La Frontera, Temuco, Chile.
} 
Desde un punto de vista morfológico, las características de clase resultan poderosamente importantes de establecer si se pretende realizar un diagnóstico de mordedura humana: es una lesión circular u ovoide que comprende dos arcos opuestos (a veces parciales), generalmente separados entre sí, donde cada arco incluye un patrón de lesiones más pequeñas representando los dientes que entraron en contacto con la superficie mordida (Bernitz et al., 2008; Forrest \& Soon). Estas pequeñas contusiones pueden presentar arrastres (poniendo en evidencia la dinámica de la mordedura) e incluso un área contusa central resultado del cierre de ambos maxilares (Fig. 1). Las huellas pueden ser únicas o múltiples, a veces superpuestas entre sí (Forrest \& Soon). En individuos vivos, las lesiones usualmente remiten en unas pocas horas o, en casos más serios, entre los 7 y los 14 días si no hubo avulsión de los tejidos. Heridas contusas y por compresión profundas pueden formar tejido cicatrizal con patrones a largo plazo muy difíciles de analizar (Dorion \& Souviron, 2011).

Estas manifestaciones morfológicas han sido destacadas como sumamente importantes para el ejercicio diagnóstico y poder diferenciarlas de otras lesiones muchas veces de apariencia similar (Dorion \& Souviron; Goodbody et al., 1976; Gold et al., 1989; Weeratna, 2014). Dorion \& Souviron afirman que, si bien en vivos el diagnóstico se ve claramente facilitado, la investigación de lesiones presentes en el cadáver debe considerar un protocolo de autopsia y un análisis morfológico apropiado de la lesión para minimizar las posibilidades de error.

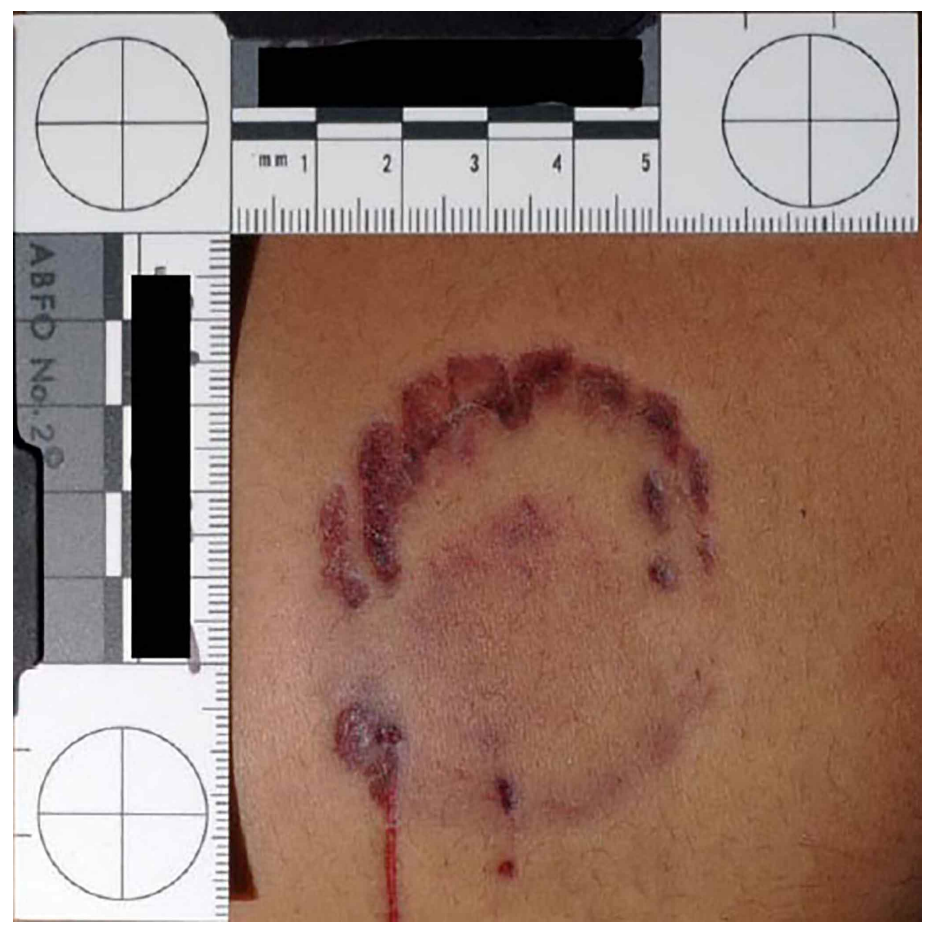

Se ha afirmado que para el ojo no entrenado, el reconocimiento de huellas por mordedura no es una tarea simple y que deben profundizarse los estudios sobre los caracteres morfológicos de estas lesiones para poder realizar diagnósticos apropiados (Weeratna). Dado que en el ámbito medicolegal la simulación experimental no es posible (por la naturaleza de los eventos analizados), y las tareas e informes se sustentan en evidencia provista por expertos (Madea, 2007), se presenta una revisión con búsqueda sistemática de documentos que reporten casos de lesiones erróneamente diagnosticadas como huellas de mordedura, se analizan sus correspondientes patrones morfológicos y se discuten sus implicancias en un correcto diagnóstico diferencial desde la perspectiva odontológica forense.

\section{MATERIAL Y MÉTODO}

Se realizó una revisión con búsqueda sistemática de la literatura siguiendo la metodología propuesta por el Instituto Joanna Briggs (Peters et al., 2015) y las directrices PRISMA (Moher et al., 2009). Las revisiones con búsqueda sistemática han sido mencionadas como la metodología apropiada si se pretende responder preguntas amplias de investigación y obtener una apreciación de la naturaleza de la evidencia disponible. Si bien estas revisiones no evalúan la calidad de los estudios incluidos (a diferencia de las revisiones sistemáticas), permiten identificar lagunas en el conocimiento, definir el alcance de futuras revisiones sistemáticas

o dirigir el desarrollo de preguntas focalizadas de investigación (Armstrong et al., 2011). Una estrategia específica de búsqueda ("bite mark" OR "bitemark") AND ("artefactual" OR "misidentification" OR "misdiagnosis" OR "mistake") fue implementada en PubMed/Medline y Google Scholar complementando con una búsqueda manual sobre las referencias identificadas, buscando documentos completos en inglés y español, los que fueron analizados de forma independiente por los dos investigadores. La búsqueda fue realizada entre los días 30 de junio y 28 de julio de 2020. Solo se incluyeron documentos sin límite de tiempo que reporten casos de lesiones en piel humana con una descripción morfológica y una causa establecida, pero erróneamente diagnosticadas como huellas de mordedura en contextos forenses. Se excluyeron estudios experimentales y documen-

Fig. 1. Típico patrón morfológico de una verdadera huella de mordedura humana (Imagen de dominio público: https:// globalwrong.files.wordpress.com/2012/09/bitemark1.jpg) 
tos que no respondieran a los objetivos. La búsqueda identificó 13 documentos (Fig. 2), cuya información relevante fue extraida y analizada según categorías específicas: tipo de documento, errores diagnósticos y sugerencias destacadas para prevenirlos. Los capítulos de libro fueron categorizados como documentos independientes en casos de autorías propias, más allá de pertenecer a un solo libro con un editor definido.
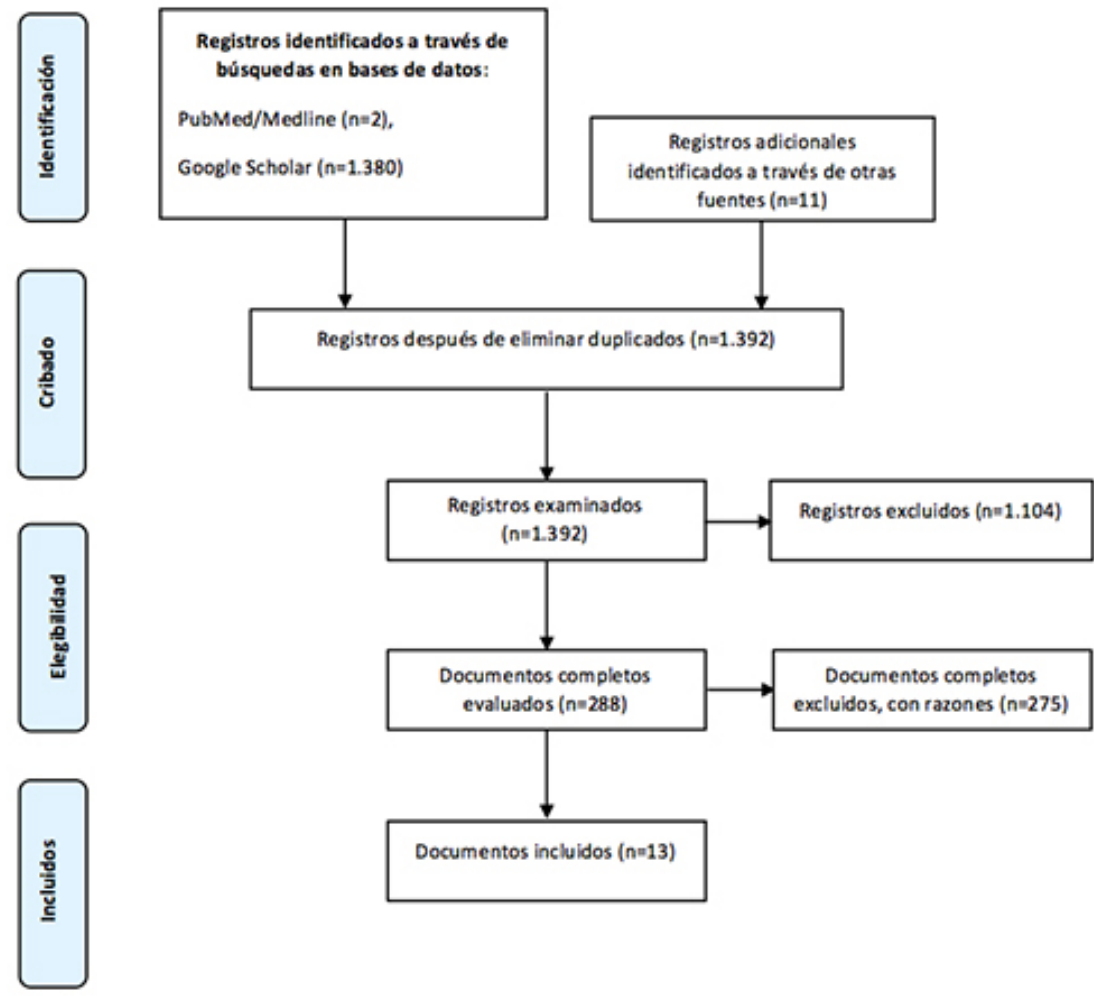

Fig. 2. Diagrama de flujo PRISMA utilizado para desarrollar la revisión con búsqueda sistemática.

\section{RESULTADOS}

Tipo de documentos y autorías. Un total de 13 documentos respondieron al objetivo de búsqueda de los cuales solo 5 fueron categorizados como artículos. Las publicaciones fueron realizadas entre los años 1989 y 2014 por un total de 17 autores (Tabla I). Es de destacar la presencia mayoritaria de autores de los EE.UU. (10/17), donde lideraron el Dr. Richard Souviron (Miami, EE.UU.) con 3 autorías y el Dr. C. Michael Bowers (Los Angeles, EE.UU.) con 2 autorías. Canadá reportó 3 autores, liderando el Dr. Robert B. J. Dorion (Montreal, EE.UU.) con 2 autorías. Debe mencionarse que el Dr. Dorion a su vez es el editor de la $2^{\mathrm{a}}$ edición del libro Bitemark Evidence. A Color Atlas and Text (2011) al cual pertenecen 4 documentos (capítulos) incluidos en esta revisión. Hubo dos autores de Australia, un autor de Reino Unido y uno de Chile, este último el Dr. Luis Ciocca Gómez citando un caso del prestigioso director del Instituto de Medicina Legal de Estrasburgo, Camille Simonin (1891-1961).

Otro dato relevante es que 12 de los 17 autores eran odontólogos, 3 médicos, 1 entomólogo y 1 antropólogo; estos dos últimos, presentando un estudio sobre descomposición e intervención de fauna cadavérica en medio marino para la detección de artefactos

Tabla I. Documentos identificados en esta revisión. ${ }^{1}$ Capítulos pertenecientes a la $2^{\circ}$ edición del libro Bitemark Evidence. A Color Atlas and Text, editado por Dorion R. B. J. (2011).

\begin{tabular}{clcc}
\hline \# Documento & Referencias Bibliográficas & País autores & Tipo de Documento \\
\hline 1 & Anders on \& Bell (2014) & Canadá & Artículo \\
2 & Bowers (2011) & EE.UU. & Capítulo \\
3 & Brooks \& Simpson (2011) & EE.UU. & Artículo \\
4 & Davis (2011) & EE.UU. & Capítulo1 \\
5 & Dorion (2011) & Canadá & Capítulo1 \\
6 & Dorion \& Souviron (2011) & Canadá, EE.UU. & Capítulo1 \\
7 & Pretty \& Bowers (2011) & Reino Unido, EE.UU. & Capítulo1 \\
8 & Senn \& Souviron (2010) & EE.UU. & Capítulo \\
9 & Ciocca Gómez (2009) & Chile & Libro \\
10 & Silver \& Souviron (2009) & EE.UU. & Libro \\
11 & James \& Cirillo (2004) & Australia & Artículo \\
12 & Sperry \& Campbell (1990) & EE.UU. & Artículo \\
13 & Grey (1989) & EE.UU. & Artículo \\
\hline
\end{tabular}


y patrones morfológicos producidos por crustáceos, citaron un reconocido caso de error diagnóstico objetivo de esta revisión (Caso \# 17 en esta revisión), última publicación detectada en la búsqueda (Anderson \& Bell, 2014).

Los documentos describieron un total de 21 casos de los cuales $19(90,48 \%$ ) acontecieron en los EE.UU. (Tabla II). Las causas definitivas fueron categorizadas en 5 tipos siguiendo las descriptivas de Dorion \& Souviron con modificaciones Ah-hoc: I) Trauma cortante o contuso por otras causas, con 10 casos reportados, 5 de ellos con heridas producidas por objetos cortantes y los 5 restantes por objetos romos; II) Condiciones médicas, tratamientos paramortem, trauma por autopsia u otros objetos, con 6 casos; III) Mordeduras animales y depredación postmortem, con 1 caso; IV) Picaduras de insectos, con 2 casos; y V) Patologías dermatológicas, con 2 casos. Al menos 3 de los casos tomaron estado público (Casos \# 10, 15 y 17), 2 de ellos con sentencia de muerte y uno con condena de 25 años a prisión perpetua. Debe mencionarse que en 2 de esos casos intervino el mismo odontólogo forense, el cual fue desacreditado como perito aun cuando una de las condenas todavía se sostiene (Balko \& Carrington, 2018).

En todos los reportes, los autores sugieren realizar un análisis minucioso del patrón morfológico, evaluar bordes, tamaño y ubicación de las lesiones así como seguir protocolos estandarizados para la realización de la autopsia, del análisis de la información de la escena, del historial médico y de los posibles tratamientos de emergencia (paramortem).

\section{DISCUSIÓN}

Los reportes de casos son considerados en las jerarquías más bajas de la evidencia científica debido a su carácter anecdótico (con la negativa categórica de muchas revistas por publicarlos); sin embargo, Madea afirma que en el ámbito medicolegal, hasta los casos más extraños pueden no ser únicos, y por su naturaleza y "peculiaridades estructurales" para resolver crímenes, son oportunidades únicas para identificar puntos débiles o sustentar estrategias probatorias en casos similares. El autor concluye que los reportes de casos en el ámbito forense pueden ayudar a identificar patrones morfológicos extraños o inusuales de lesiones y establecer nuevos criterios para aportar prueba pericial. Por su parte, Senn \& Souviron (2010), afirman que estudiar casos controvertidos es una manera excelente de aprender y revisar permanentemente los estándares y factores que contribuyeron a su desarrollo y discusión. Probablemente especulativo, pero la ausencia evidente en revistas especializadas de reportes de errores diagnósticos de huellas de morde- dura tomando en consideración el aparato mediático alrededor de esta evidencia (Fonseca, 2020) es una señal desafortunada de subestimación del problema, de corporativismo de la especialidad o simplemente de desinterés editorial. Si bien los 13 documentos podrían dar cuenta de una visibilidad, al menos mínima del fenómeno, 4 de ellos son capítulos de un mismo libro (un claro referente por la confluencia de autores expertos en el tópico), y solo 5 son publicaciones en revistas indexadas. Tampoco es coincidente que la mayor afluencia de documentos se presente como reacción (explícita en algunos casos) al controversial pero "seminal" reporte de la Academia Nacional de Ciencias de los Estados Unidos, Strengthening Forensic Science in the United States: A Path Forward (Committee on Identifying the Needs of the Forensic Science Community, 2009). Este documento, luego de analizar el rol de los odontólogos forenses y de las bases científicas del análisis de huellas de mordedura, pone un manto de dudas en el valor y confiabilidad de esta evidencia para identificar a un criminal (Fonseca et al., 2013). El reporte afirma que aún utilizando las recomendaciones del American Board of Forensic Odontology (ABFO) -ente reconocido mundialmente en la especialidad por sus contribuciones y actualización de protocolos-, las grandes diferencias de criterio y opinión al hacer los análisis, llevan peligrosamente a igualmente altos niveles de diagnósticos erróneos (Committee on Identifying the Needs of the Forensic Science Community). Así mismo, ese reporte asume como "razonable" la confiabilidad del método para excluir sospechosos, algo que el Dr. Richard Souviron retoma casi de forma permanente (Souviron \& Haller). Probablemente desde el ensayo de "educar a través del error", Souviron -en colaboración- reporta 13 de los 21 casos identificados, con participaciones protagónicas como coautor en un libro (Silver \& Souviron, 2009), como coautor de un capítulo en otro (Senn \& Souviron) y finalmente como coautor de un capítulo completo dedicado exclusivamente a este problema (Dorion \& Souviron). Debe mencionarse que Souviron, diplomado y Presidente de ABFO en el periodo 1987-1988, ha sido Jefe Odontólogo Forense para el Condado de Dade en Florida (EE.UU.) y ha participado en casi un centenar de casos tanto para la defensa como para la fiscalía, incluyendo el reconocido caso de Ted Bundy (Fonseca et al.). Claramente en los EE.UU. el fenómeno de la identificación errónea al utilizar como evidencia las huellas de mordedura ha significado un verdadero dolor de cabeza para ABFO y los odontólogos forenses (Fonseca et al.). Así mismo, este fenómeno ha resignificado la conveniente fundamentación científica en ese país y en todo el mundo para este tipo de pruebas, como así lo demuestra la nueva investigación desarrollada en Australia buscando clarificar métodos y alcances de los informes (Forrest \& Soon).

El diagnóstico de una lesión en un contexto 


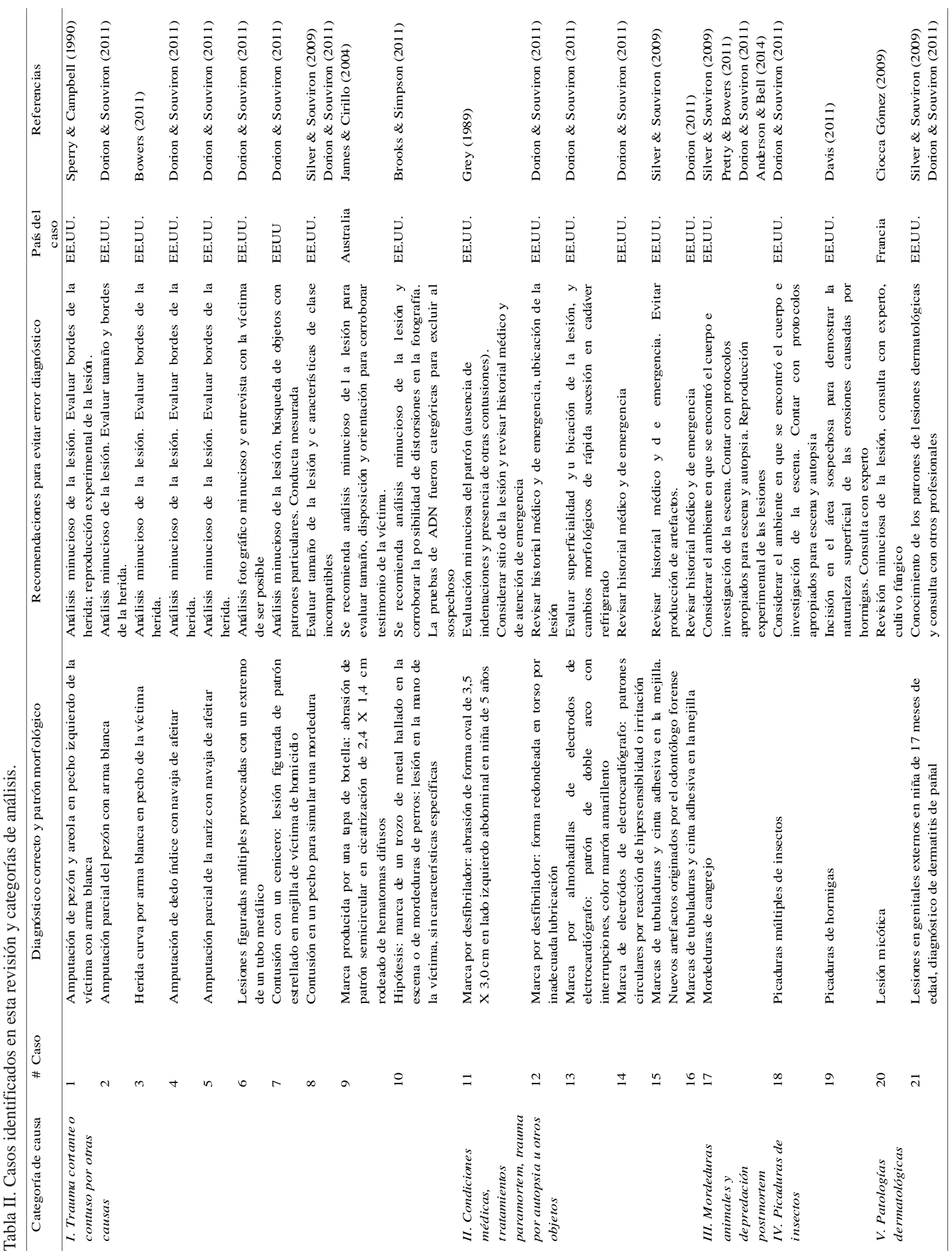


medicolegal se fundamenta en la anamnesis, el examen físico y el conocimiento necesario de las características morfológicas de las lesiones para poder identificarlas, entendiendo que ellas responden directamente al mecanismo y objeto que las produjo (Bórquez). Tomando en consideración que las lesiones pueden ser producidas por agentes biológicos, químicos o físicos -y dentro de estos últimos los agentes mecánicos-, el tamaño, forma, bordes, profundidad, puentes tisulares, presencia de partículas externas, vitalidad, etc. son todos elementos morfológicos en esas lesiones que deben ser cuidadosamente explorados y correlacionados con las circunstancias para poder realizar un diagnóstico certero (Bórquez). Silver \& Souviron llaman la atención respecto de que muchos de los diagnósticos presuntivos iniciales pueden haber sido realizados por socorristas, criminalistas, enfermeros o médicos sin conocimiento, pero condicionando absolutamente las conductas (técnicas e incluso jurídicas) posteriores.

Particularmente los odontólogos deben ser cuidadosos y circunspectos en las evaluaciones de todas las lesiones, pero especialmente en las producidas por mordeduras: debe conocer las circunstancias del evento, revisar fotografías de la escena, conocer de posibles intervenciones médicas, información de hora, lugar, temperatura, probabilidad de intervención de insectos o animales, así como también de declaraciones de testigos presenciales (Silver \& Souviron). Entendidas las huellas de mordedura como lesiones figuradas producidas por cuerpos romos (los dientes), el análisis diagnóstico debe ser mucho más que solo comparar o hacer coincidir un patrón morfológico en la lesión con una dentadura sospechosa, y que el proceso debe iniciarse respondiendo a la pregunta básica y más difícil: “¿es o no una huella de mordedura?” (Souviron \& Haller). Reesu \& Brown aseguran que el principio para identificar una lesión como huella de mordedura es complejo pues existen muchos factores que necesitan ser considerados: el lugar donde se encuentra la lesión, la movilidad y elasticidad tisular, la profundidad y naturaleza de las estructuras tisulares subyacentes, el historial médico de la persona lesionada, la antigüedad de la lesión y el grado de trauma. Dado que son necesarias fotografías de la supuesta huella, estas deben ser supervisadas en su registro por odontólogos forenses o por fotógrafos con la experiencia y conocimiento necesario para producir una imagen útil al análisis forense posterior. Los autores, aseveran que las diferencias de opinión entre odontólogos forenses respecto a las huellas de mordedura no solo abarcan inconsistencias en la posibilidad de identificar al perpetrador, sino incluso a la certeza de diagnosticar si la lesión es o no una huella de mordedura (Reesu \& Brown). Silver \& Souviron recomiendan al odontólogo una conducta sumamente cautelosa: si no puede ser fácilmente reconocida como una huella de mordedura, una actitud pru- dente sería informar "no sé qué la causó" o simplemente “son necesarios más análisis para poder dar una opinión”.

Una evaluación adecuada de las huellas de mordedura posee dos factores contingentes: a) en primer lugar, el médico forense que realiza la autopsia debe reconocerla correctamente o excluirla como tal, tomando en consideración que el patrón morfológico típico puede no estar siempre presente, que puede pasar por alto formas atípicas de huella de mordedura, y que existen lesiones producidas por otras causas que poseen gran similitud morfológica; b) el segundo factor contingente recae en el odontólogo quien, aún con experiencia, puede perpetuar el diagnóstico erróneo dado por el médico forense o incluso por el mismo personal policial o criminalístico. Una mal etiquetada "huella de mordedura" puede seguir este curso originando una evidencia falaz y una potencial identificación errónea de un sospechoso (Sperry \& Campbell, 1990).

Cuando una lesión es evaluada inicialmente considerando la posiblidad de que su origen sea una mordedura humana, la primera pregunta a ser respondida debería ser “es verdaderamente una lesión por mordedura?” (Sperry \& Campbell). Identificarla como tal debería ser el primero (y fundamental) paso a seguir en un protocolo de análisis lesionológico (Bernitz et al.) tomando en cuenta la posibilidad de que otros agentes causales puedan originar patrones morfológicos de gran similitud con los producidos por dientes humanos. Considerando los casos recabados en esta revisión, se proponen las siguientes categorías de causas confirmadas como susceptibles de error diagnóstico como huellas de mordedura (Tabla II):

I. Trauma cortante o contuso por otras causas. Dorion \& Souviron, afirman que pueden producirse lesiones figuradas por efecto traumático o compresión pasiva de objetos, en algunos casos de gran similitud a huellas de mordedura. Los objetos pueden producir patrones morfológicos ovales, incluso con características de clase que al ojo desprevenido puede fácilmente llevarlo a una malinterpretación. Dorion (2011) menciona que en víctimas vivas siempre que sea posible, deben tomarse declaraciones para abordar la probable etiología: patrones repetitivos de golpes con objetos cuyas características de clase son similares a la dentición humana, pueden también ser fácilmente confundibles con huellas de mordedura (Dorion \& Souviron). Sin embargo, los autores afirman que, aunque disponible, el testimonio de las víctimas puede no ser necesariamente confiable. Según diferentes autores, marcas de cascos de animales (caballos y vacas), de talones en pisotones (más aún si el calzado posee texturas similares a dientes), joyas circulares (anillos), hebillas de cinturón, mangos de linterna o juguetes infanti- 
les pueden todos ser objetos potencialmente productores de lesiones similares a huellas de mordedura, incluso generadas ex profeso por la víctima para simular agresiones (Bowers, 2011; Dorion \& Souviron; Silver \& Souviron). La posiblidad de error se potenciará aún más si el análisis solo se apoya en una interpretación fotográfica sin escalimetría, sin impresiones ni modelos, sin conservación de tejidos, transiluminación o histología, en palabras de los autores, sin adherir a un protocolo estandarizado (Dorion \& Souviron).

En el caso de las heridas cortantes (producidas por objetos filosos), cuando aparecen en zonas comúnmente asociadas con mordeduras humanas (pechos, nalgas, etc.) la distorsión originada por la elasticidad propia de los tejidos puede llevar a errores diagnósticos. En la mayoría de las huellas de mordedura, aun producidas en las partes más blandas de la anatomía, los mecanismos de producción son esencialmente una mezcla de abrasiones y contusiones, y en el menor de los casos, laceraciones profundas o arrancamientos. Los dientes humanos, al ser objetos de bordes romos o redondeados, no tienen la posiblidad de actuar como tijeras lo que se suma a la resistencia y elasticidad de la piel humana por lo que la evaluación minuciosa de bordes netos y la ausencia de puentes tisulares facilitaría enormemente diferenciarlos de heridas cortantes por acción de cuchillos, navajas, etc. (Dorion \& Souviron; Grey, 1989). Si bien la curvatura de un corte con arma blanca puede llamar a confusión, debería comprobarse si el ancho es compatible con el del arco de una dentadura humana (Bowers).

\section{Condiciones médicas, tratamientos paramortem, trau-} ma por autopsia u otros objetos. Una de las circunstancias clásicas de error diagnóstico de huellas de mordedura, son las marcas resultantes de tratamientos médicos de emergencia, intubaciones o cintas adhesivas (Dorion; Silver \& Souviron). El caso reportado por Bernstein (citado por Dorion \& Souviron) expone la probabilidad de generar patrones figurados por quemadura con un defibrilador, si las palas no han sido apropiadamente lubricadas. De no contarse con fotografías de la escena debe actuarse con suma precaución ya que muchas víctimas recibiendo atención médica de emergencia, pueden presentar lesiones y patrones morfológicos de trauma como resultado de ese tratamiento (Dorion \& Souviron; Silver \& Souviron). En mesa de morgue, la información precisa de los eventos que conducen a la autopsia podrá prevenir de una mala interpretación de esos patrones. Los autores, citando una comunación personal de Vale \& Dorion, relatan el caso de una lesión muy similar a una huella de mordedura en varios aspectos: dos arcos enfrentados con patrones figurados interrumpidos que sugerían marcas individuales de dientes. Sin embargo, el color amarillento-amarronado de la lesión era incompatible con el color rojo intenso o azul-violáceo característico de una contusión perimortem o antemortem reciente. En interconsulta con dos odontólogos forenses, una nueva observación de la lesión apenas 24 hs después y con el cadáver refrigerado, dejó en evidencia un cambio sustancial en el patrón morfológico que incompatibilizó aún más la posibilidad de ser una huella de mordedura humana. Dado que la lesión se localizó en un área comúnmente utilizada para colocar las almohadillas del monitor del electrocardiógrafo, se realizó la consulta al personal de emergencia el cual confirmó el uso de este dispositivo. Las condiciones en que la almohadilla fue colocada y las circunstancias de esa atención explicaron las características morfológicas de la lesión, diagnosticada finalmente como una abrasión superficial con cambios en su patrón por acción de la refrigeración y deshidratación del tejido (Dorion \& Souviron). Los autores afirman que los electrodos del electrocardiógrafo pueden producir irritaciones o reacción de hipersensiblidad con imágenes muy similares a mordeduras.

\section{Mordeduras de animales y depredación postmortem.}

En la mayoría de los casos de mordeduras animales no fatales, si la víctima está en condiciones hacerlo, puede simplemente describir las circunstancias e identificar al animal como causante de las lesiones (Souviron, 2011). En mordeduras fatales, es el médico forense el encargado de diagnosticar y consultar con un odontólogo forense si las lesiones figuradas sospechosas son consistentes con huellas de mordedura humana o animal. El odontólogo forense examinará, registrará, documentará y preservará la lesión según sea necesario (Souviron). Para Dorion \& Souviron, la depredación postmortem es la segunda causa más común de lesiones figuradas. La posiblidad de error diagnóstico es aún mayor si la víctima se encuentra en descomposición, por lo que la evaluación de la escena y las circunstancias son factores fundamentales para la interpretación de la lesión figurada, especialmente si el cadáver fue hallado al aire libre o en presencia de animales (Bowers; Souviron). Cuando el cadáver ha quedado en el agua, deben esperarse marcas por intervención postmortem de peces, cangrejos, tortugas, etc. (Silver \& Souviron). En el caso de la acción de mamíferos, según Dorion \& Souviron, una distancia de más de $40 \mathrm{~mm}$ entre caninos en el mismo arco debería ser pista suficiente del origen no humano de las lesiones, por lo que se acentúa la necesidad de un enfoque minucioso y cauteloso al interpretar su morfología. Kashyap et al. (2015) en un estudio experimental, aseguran que mientras esa distancia en humanos va de 36 a $42 \mathrm{~mm}$ en hombres y de 34 a 41 $\mathrm{mm}$ en mujeres con una forma de arco predominantemente oval y morfología rectangular de incisivos y trapezoidal de caninos, en perros la distancia intercanina va de 36 a $44 \mathrm{~mm}$ maxilar, 28 a $29 \mathrm{~mm}$ mandibular con un tamaño de arco variable según la raza, forma de predominio circular-ovalado y morfología similar de incisivos y caninos. 
IV. Picaduras de insectos. La actividad postmortem de insectos es el más común de los patrones lesionológicos presentes en cadáveres recuperados del exterior (Dorion \& Souviron; Silver \& Souviron). La actividad de los insectos comienza minutos después de producida la muerte y se ve influenciada por la temperatura, la humedad, el viento, el suelo, las bacterias, la posición corporal y las circunstancias de muerte, entre otros factores. Hormigas, cucarachas e insectos desconocidos han sido reportados como productores de erosiones y patrones muy similares a huellas de mordedura humanas, sorprendentemente utilizados en algunos casos para enviar individuos a prisión (Silver \& Souviron). Aunque el patrón arciforme de una huella de mordedura conformaría el rasgo morfológico característico, existen huellas de mordedura con patrones múltiples superpuestos, e incluso marcas de insectos siguiendo una forma de arco simple lo que fácilmente llevaría a la confusión (Dorion \& Souviron; Souviron). Para Dorion \& Souviron, seguir protocolos estrictos tanto en la escena como durante la autopsia podría resolver rápidamente el problema. Bowers afirma que en picaduras de insectos, la aparición inusualmente repetitiva de patrones o la ausencia de hematomas subyacentes bajo la dermis son elementos morfológicos a tomar en consideración para realizar los diagnósticos diferenciales. Una incisión en el área sospechosa puede demostrar la naturaleza superficial de las erosiones cuando han sido causadas por insectos; la opinión de un experto siempre será recomendable (Davis, 2011; Dorion \& Souviron; Senn \& Souviron; Silver \& Souviron). Silver \& Souviron recomiendan contar con una documentación fotográfica completa de la escena mostrando el cadáver, el área circundante y los patrones de lesiones antes y después de la manipulación (con y sin escalimetría) pues puede ser de gran ayuda para explicar patrones morfológicos producidos por insectos (especialmente hormigas).

V. Patologías dermatológicas. Existe una gran gama de enfermedades y condiciones patológicas dérmicas que pueden producir lesiones anulares erosivas o eritematosas fácilmente confundibles con huellas de mordedura: tinea circinata, impético, eritema polimorfo, eritema anular, granuloma anular, pitiriasis rosas, líquen plano, alergias de contacto, urticaria, herpes, y en niños dermatitis del pañal son algunos de los ejemplos más citados (Dorion \& Souviron; Silver \& Souviron). La interconsulta con otros odontólogos y con especialistas puede ayudar a resolver el diagnóstico (Dorion \& Souviron). Simonin (citado por Ciocca Gómez, 2010), relata el caso de un individuo con una aparente huella de mordedura en su mejilla, a la que culpaba de la violencia con la que había matado al supuesto mordedor. La opinión experta puso en evidencia que la lesión en realidad era de origen micótico.
Casos, errores y consecuencias. Diferentes autores han reportado casos en los que una verdadera huella de mordedura había sido inicialmente confundida con lesiones dermatológicas (Gold et al.; Weeratna) o con heridas producidas por otros objetos (Goodbody et al.). Sin embargo, el sentido inverso -y desafortunado- de diagnosticar como huella de mordedura una lesión de otra causa, o peor aún, asignarle valor identificatorio y llevar a un individuo injustamente a prisión a partir de ello, posee a las claras una significación catastrófica al sistema. En 1995, Kennedy Brewer fue condenado a muerte por violación y asesinato gracias a que 19 huellas de mordedura en el cuerpo de una niña de 3 años, fueron asociadas "fuera de toda duda" con su dentadura por un odontólogo forense (Caso \# 17 en esta revisión). El Dr. Richard Souviron, odontólogo forense por la defensa, testificó que las lesiones ni siquiera eran mordeduras humanas: "Podría ser actividad de insectos, peces, tortugas o quién sabe qué". Esa misma afirmación fue ratificada por otros cuatro peritos (tres odontólogos y un entomólogo) luego de replicar esas lesiones en cerdos observando la actividad postmortem de cangrejos. Sin embargo, debieron pasar 15 años para que pruebas genéticas demostraran la inocencia de Brewer y lograran su exoneración (Anderson \& Bell; Fonseca et al.; Senn, 2011; Senn \& Souviron; Silver \& Souviron; The National Registry of Exonerations, 2020b). Silver \& Souviron, respecto al caso, encuentran fuera de todo sentido común el diagnóstico de esas 19 lesiones como "huellas de mordedura" cuando supuestamente solo se veían marcas de los dos incisivos centrales superiores y ninguna marca de los incisivos inferiores... “¿Cómo pueden hacerse 19 mordeduras solo con dos o tres incisivos superiores y sin marcas de los incisivos inferiores? ¡Una locura!” (sic). Giannelli, afirma que un sorprendente número de casos han involucrado desacuerdos en los que mientras la fiscalía hace identificaciones positivas a partir de una huella, peritos de la defensa testifican que la marca ni siquiera es una huella de mordedura (ejemplificando esta afirmación, Giannelli cita los casos de Harrison v. State y People v. Smith, en este último con una discrepancia de opiniones de cuatro peritos por la fiscalía por un lado, y tres por la defensa por el otro lado).

El caso de Anthony Keko en Lousiana (EE.UU.), cubierto incluso por la prensa internacional, también revistió ribetes de sensacionalismo y mala fortuna: fue condenado por el homicidio de su esposa con una única vinculación de violencia en una supuesta huella de mordedura en el hombro izquierdo de la víctima. Según relatan Silver \& Souviron, más allá de lo cuestionable de la técnica de análisis, lo más increíble fue que había ausencia absoluta de marcas de los incisivos superiores o inferiores y solo las había del canino inferior derecho y de los premolares. Aunque los peritos de la defensa argumentaron que el patrón 
morfológico no se correspondía de ninguna manera con una huella de mordedura y que anatómicamente era imposible que se hubiera producido de la manera en que se había informado, Anthony Keko fue condenado a cadena perpetua. Tras diversas apelaciones y la falta de credibilidad del perito de la fiscalía, finalmente Keko fue exonerado (Balko \& Carrington; Silver \& Souviron; The National Registry of Exonerations, 2020a).

En agosto de 1993, Pamela Richards fue salvajemente golpeada, estrangulada y finalmente ultimada al aplastársele el cráneo con un bloque de hormigón (Gianelli, 1993). Su marido, William Richards, fue condenado en 1997 de 25 años a prisión perpetua por el crimen, sobre la base de una supuesta huella de mordedura en la mano derecha de la víctima, informada por un reconocido odontólogo forense como coincidente con la particular dentadura del acusado (caso \# 10 en esta revisión). La investigación de la escena fue realizada tres horas después (con la consiguiente contaminación), y la pericia sobre la "huella de mordedura" fue realizada utilizando fotografías de muy baja calidad tomadas durante la autopsia. Nuevas investigaciones posteriores revelaron que la marca en la mano se correspondía con un trozo de metal hallado en la escena; otros expertos concluyeron que las marcas podían corresponder con mordeduras de perros (presentes y con demostrada afectación de la escena). De hecho, en un nuevo juicio el mismo odontólogo forense que identificó a William Richards se retractó de su informe original aseverando que "no podía decir con certeza que la lesión en la mano de la víctima sea una lesión por mordedura humana". Debieron pasar 19 años para que las pruebas genéticas excluyeran a William Richards de la escena y lo exoneraran finalmente en 2016 (Brooks \& Simpson, 2011; The National Registry of Exonerations, 2020c).

Silver \& Souviron relatan un caso de lesiones por intervenciones hospitalarias evaluadas por un odontólogo sin conocimiento de las circunstancias del suceso. Jimmie Duncan fue condenado en 1998 a muerte sobre la base de una huella de mordedura en la mejilla derecha de una niña de 5 años ahogada en la bañera (Caso \# 15 en esta revisión). Si bien el odontólogo forense informó que esa marca se correspondía sin dudas con los dientes del acusado, nunca tomó conocimiento de las maniobras hospitalarias de reanimación que se realizaron sobre la víctima antes de fallecer y que, de hecho, las marcas en la cara tenían su origen en la cinta adhesiva colocada para sujetar las tubuladuras, luego retirada para la autopsia. Aun comprobado que el odontólogo forense generó artefactos en la mejilla de la niña al presionar repetidas veces un modelo dental del acusado, Duncan todavía se encuentra en el "pasillo de la muerte" esperando su ejecución (Balko \& Carrington).
Bernstein (2011) afirma que incluso lesiones similares a huellas de mordedura deben ser documentadas y analizadas en profundidad por un odontólogo forense, ya que el ignorar tal lesión por ser supuestamente atribuida a alguna otra causa, podría ser capitalizado por la contraparte como una huella de mordedura no coincidente con la dentición del acusado. Una lesión figurada puede contener elementos morfológicos suficientes para ser diagnosticada como huella de mordedura, puede apenas presentarlos o simplemente no serlo; en cualquier caso, el odontólogo forense debe tener la oportunidad de examinar esos patrones morfológicos lesionales y juzgar su relevancia. Kaliszan et al. (2011), sugieren que en muchos casos, el reproducir experimentalmente la potencial lesión puede posibilitar confirmar o excluir determinados agentes causales (objetos particularmente) y evitar la malinterpretación del origen de la lesión.

Davis menciona que el problema es que los médicos forenses o los mismos odontólogos pueden no poseer adecuados conocimientos y experiencia, por lo que es prudente contar con un par de ojos cualificados para evaluar estas lesiones. Los profesionales involucrados pueden contribuir con decisiones o conclusiones inexactas si no se ha prestado atención detallada durante el examen y registro de las lesiones o si no se poseen conocimientos suficientes sobre el tema, afectando las fases interpretativas y jurídicas posteriores (Zeyfeog ${ }^{\top}$ lu et al., 2010). En 2017, Bernitz y Kloppers reportan un interesante caso en Sudáfrica destacando el hecho de que solo expertos en huellas de mordedura deberían ser quienes las examinen: un patólogo forense equivocó su declaración al centrar su evaluación incorrectamente en un grupo de lesiones que efectivamente no eran una huella de mordedura, cuando la lesión a diagnosticar estaba exactamente al lado en la fotografía. Los autores relatan: “i(El patólogo forense) estaba absolutamente en lo cierto, ya que no estaba describiendo el conjunto correcto de marcas! El juez y el abogado del estado se sintieron muy aliviados cuando les mostramos que las marcas señaladas por el patólogo forense no eran en realidad las huellas de mordedura infligidas por el sospechoso, sino que estaban más atrás y el patólogo las había pasado por alto". Las lesiones malinterpretadas por el patólogo forense en realidad eran solo artefactos postmortem.

Dorion \& Souviron afirman que, al momento de analizar durante la autopsia un patrón morfológico lesional, es importante obtener fotografías de la escena y estar bien informado de las circunstancias que llevaron a la muerte al individuo, así como de las posibles intervenciones de socorristas o del mismo procedimiento autópsico. Sin un conocimiento completo u otra información pertinente relacionada con el patrón lesionológico (incluyendo el historial médico), puede producirse un error diagnóstico, y este lle- 
var a conclusiones trágicas. Los informes deben ser emitidos cautelosamente, siempre buscando segundas opiniones independientes, y siendo conservadores antes de dar una opinión probatoria (Silver \& Souviron).

Pretty \& Bowers (2011) aseveran que los diagnósticos erróneos son generalmente atribuibles a la escasa cualificación o deficiente mirada científica de algunos odontólogos, y que esto no debería afectar las bases científicas de la ciencia en sí, sino más bien su práctica. Page et al. (2011) afirman que los errores diagnósticos y las identificaciones equívocas no son resultado de que dos personas tengan una misma huella, sino producto de las especulaciones, de la falta de estándares y protocolos. El reconocimiento de patrones morfológicos adecuados, la observación cuidadosa, consensuada, objetiva y cualificada al menos dará oportunidad a la mesura y al sano juicio. Citando a Moritz, Dorion \& Souviron afirman: "Si la evidencia se ha recopilado y preservado adecuadamente, un error de interpretación siempre puede ser corregido. Si no se conservan los hechos necesarios para corregir esa interpretación, el error es irreversible".

DAL SANTO-MENDOZA, F. \& FONSECA, G. M. Morphological patterns misdiagnosed as bite marks in forensic contexts: a scoping review. Int. J. Morphol., 39(2):642-652, 2021.

SUMMARY: Bite mark analysis for forensic identification can be key to establishing the innocence or guilt of the defendant. However, this evidence has been challenged for its lack of consensus and objectivity, and its reported misidentifications. We present a scoping review of cases indicating misidentifications and their characterizations, and recommendations for avoid them are discussed. The strategy ("bite mark" OR "bitemark") AND ("artifactual" OR "misidentification" OR "misdiagnosis" OR "mistake") was used, including cases of human bite marks on skin with reported misdiagnosis, excluding experimental designs, bite marks on food or objects. A total of 13 documents were selected reporting 21 cases, covering the years 1989 to 2014. The morphological patterns with the highest report of misdiagnosis were those produced by cutting or blunt trauma due to other causes (10 cases), followed by those produced by medical conditions and emergency treatments ( 6 cases). At least three of the cases became public, two of them with a death sentence, and one with a 25-year sentence to life in prison. The thoroughness and standardization of the morphological evaluation of this type of evidence has been emphasized due to the significant number of erroneous convictions. This review highlights the scarce exposure and analysis of these errors, with insufficient information for critical meaningful learning and the design of tools to avoid them. There is agreement on the need to standardize and optimize protocols for these evidences and adopt a reflective behavior for their analysis for forensic identification purposes. dentistry.

\section{REFERENCIAS BIBLIOGRÁFICAS}

Anderson, G. S. \& Bell, L. S. Deep coastal marine taphonomy: investigation into carcass decomposition in the saanich inlet, British Columbia using a baited camera. PLoS One, 9(10):e110710, 2014.

American Board of Forensic Odontology (ABFO). ABFO Standards and Guidelines for Evaluating Bitemarks. American Board of Forensic Odontology, 2018. Disponible en: http://abfo.org/wp-content/uploads/ 2012/08/ABFO-Standards-Guidelines-for-Evaluating-Bitemarks-Feb2018.pdf

Armstrong, R.; Hall, B. J.; Doyle, J. \& Waters, E. Cochrane update. 'Scoping the scope' of a Cochrane review. J. Public Health (Oxf.), 33(1):147-50, 2011.

Balko, R. \& Carrington, T. The cadaver king and the country dentist. New York, PublicAffairs, 2018.

Bernitz, H.; Owen, J. H.; van Heerden, W. F. \& Solheim, T. An integrated technique for the analysis of skin bite marks. J. Forensic Sci., 53(1):194-8, 2008.

Bernstein, M. L. Reconstructive Bitemark Analysis. En: Dorion, R. B. J. (Ed.). Bitemark Evidence. A Color Atlas and Text. 2nd ed. Boca Raton, CRC Press, 2011.

Bórquez, P. Elaboración del informe médico de lesiones. Rev. Med. Chile, 140:386-9, 2012

Bowers, C. M. Recognition, Documentation, Evidence Collection, and Interpretation of Bitemark Evidence. En: Bowers, C. M. (Ed.). Forensic Dental Evidence: An Investigator's Handbook. 2nd ed. San Diego, Elsevier, 2011.

Brooks, J. \& Simpson, A. Blood sugar sex magik: a review of postconviction DNA testing statutes and legislative recommendations. Drake L. Rev., 59(3):799-866, 2011.

Ciocca Gómez, L. Odontología Médico-Legal. Aspectos Forenses, Profesionales y Sociales. Santiago, Ediciones Jurídicas de Santiago, 2010.

Committee on Identifying the Needs of the Forensic Science Community; Committee on Science, Technology \& Law Policy and Global Affairs, Committee on Applied and Theoretical Statistics, Division on Engineering and Physical Sciences. National Research Council of the National Academies. Strengthening Forensic Science in the United States: A Path Forward. Washington DC, The National Academies Press, 2009.

Davis, J. H. Role of the Medical Examiner, Coroner, and Pathologist. En: Dorion, R. B. J. (Ed.). Bitemark Evidence. A Color Atlas and Text. 2a ed. Boca Raton, CRC Press, 2011.

Dorion, R. B. J. Human bitemarks. En: Dorion, R. B. J. (Ed.). Bitemark Evidence. A Color Atlas and Text. 2a ed. Boca Raton, CRC Press, 2011.

Dorion, R. B. J. \& Souviron RR. Patterns, Lesions, and Trauma-Mimicking Bitemarks. En: Dorion, R. B. J. (Ed.). Bitemark Evidence. A Color Atlas and Text. 2a ed. Boca Raton, CRC Press, 2011.

Fonseca, G. M. Forensic Dentistry: Far beyond Netflix. J. Forensic Leg. Med., 74:102032, 2020.

Fonseca, G. M.; Briem-Stamm, A. D.; Cantín, M.; Lucena, J. \& Bentkovski, A. Forensic dentistry I: bite marks. Int. J. Odontostomatol., 7(1):14957, 2013.

Forrest, A. \& Soon, A. Bite Marks. En: Taylor, J. A. \& Kieser, J. A. (Eds.). Forensic Odontology: Principles and Practice. Chichester, Wiley Blackwell, 2016.

Gianelli, P. C. Junk science: The criminal cases. J. Crim. Law Criminol., 84(1):105-28, 1993.

Gold, M. H.; Roenigk, H. H.; Smith, E. S. \& Pierce, L. J. Human bite marks. Differential diagnosis. Clin. Pediatr. (Phila.), 28(7):329-31, 1989.

Goodbody, R. A.; Turner, C. H. \& Turner, J. L. The differentiation of toothed marks: report of a case of special forensic interest. Med. Sci. Law, 16(1):44-8, 1976. 
Grey, T. C. Defibrillator injury suggesting bite mark. Am. J. Forensic Med. Pathol., 10(2):144-5, 1989.

James, H. \& Cirillo, G. N. Bite mark or bottle top? J. Forensic Sci., 49(1):119-21, 2004

Kaliszan, M.; Karnecki, K.; Akçan, R. \& Jankowski, Z. Striated abrasions from a knife with non-serrated blade--identification of the instrument of crime on the basis of an experiment with material evidence. Int. J. Legal Med., 125(5):745-8, 2011.

Kashyap, B.; Anand, S.; Reddy, S.; Sahukar, S. B.; Supriya, N. \& Pasupuleti, S. Comparison of the bite mark pattern and intercanine distance between humans and dogs. J. Forensic Dent. Sci., 7(3):1759, 2015.

Moher, D.; Liberati, A ; Tetzlaff, J. \& Altman, D. G. Preferred reporting items for systematic reviews and meta-analyses: the PRISMA statement. Ann. Intern. Med., 151(4):264-9, 2009.

Page, M.; Taylor, J. \& Blenkin, M. Uniqueness in the forensic identification sciences--fact or fiction? Forensic Sci. Int., 206(1-3):12-8, 2011.

Peters, M. D. J.; Godfrey, C. M.; McInerney, P.; Baldini Soares, C.; Khalil, H. \& Parker, D. The Joanna Briggs Institute Reviewers' Manual 2015: Methodology for JBI Scoping Reviews. Adelaide, The Joanna Briggs Institute, 2015.

Pretty, I. A. \& Bowers, C. M. Wrongful Convictions and Erroneous Bitemark Opinions. En: Dorion, R. B. J. (Ed.). Bitemark Evidence. A Color Atlas and Text. 2a ed. Boca Raton, CRC Press, 2011.

Reesu, G. V. \& Brown, N. L. Inconsistency in opinions of forensic odontologists when considering bite mark evidence. Forensic Sci. Int., 266:263-70, 2016.

Senn, D. R. History of Bitemark Evidence. En: Dorion, R. B. J. (Ed.). Bitemark Evidence. A Color Atlas and Text. 2a ed. Boca Raton, CRC Press, 2011.

Senn, D. R. \& Souviron, R. R. Bitemarks. En: Senn, D. R. \& Stimson, P. G (Eds.). Forensic Dentistry. 2a ed. Boca Raton, CRC Press, 2010.

Silver, W. E. \& Souviron, R. R. Dental Autopsy. Boca Raton, CRC Press, 2009.

Souviron, R. R. Animal bites. En: Dorion, R. B. J. (Ed.). Bitemark Evidence. A Color Atlas and Text. 2a ed. Boca Raton, CRC Press, 2011.

Souviron, R. \& Haller, L. Bite mark evidence: Bite mark analysis is not the same as bite mark comparison or matching or identification. $J$. Law Biosci., 4(3):617-22, 2017.

Sperry, K. \& Campbell, H. R. Jr. An elliptical incised wound of the breast misinterpreted as a bite injury. J. Forensic Sci., 35(5):1226-35, 1990.

The National Registry of Exonerations. Anthony Keko, 2020a. Disponible en: https://www.law.umich.edu/special/exoneration/Pages/ casedetail.aspx? caseid $=4203$

The National Registry of Exonerations. Kennedy Brewer, 2020b. Disponible en: https://www.law.umich.edu/special/exoneration/Pages/ casedetail. aspx ? caseid $=3047$

The National Registry of Exonerations. William Richards, 2020c. Disponible en: https://www.law.umich.edu/special/exoneration/Pages/ casedetail. aspx ?caseid $=4929$

Weeratna, J. B. Are they dermatological lesions, bottle top burns or bite mark injuries? J. Forensic Odontostomatol., 32(1):1-8, 2014.

Zeyfeog ${ }^{\natural}$ lu, Y.; Uluçay, T.; Yavuz, M. S. \& As, irdizer, M. Incorrect identification in forensic medicine (wrong conclusion): a case report. Ulus Travma Acil Cerrahi Derg., 16(2):185-8, 2010.

\author{
Dirección para correspondencia: \\ Dr. Gabriel M. Fonseca \\ Centro de Investigación en Odontología Legal y Forense \\ $(\mathrm{ClO})$ \\ Facultad de Odontología \\ Universidad de La Frontera \\ Francisco Salazar 01145 \\ Temuco - CHILE
}

E-mail: gabriel.fonseca@ufrontera.cl

Recibido : 22-12-2020

Aceptado: 19-01-2021 